\title{
Linearized comparison criteria for a nonlinear neutral differential equation
}

\author{
by XINPIng GuAn (Qiqihar), Jun YANG (Qiqihar) and \\ Sui Sun Cheng (Hsinchu)
}

\begin{abstract}
A class of nonlinear neutral differential equations with variable coefficients and delays is considered. Conditions for the existence of eventually positive solutions are obtained which extend some of the criteria existing in the literature. In particular, a linearized comparison theorem is obtained which establishes a connection between our nonlinear equations and a class of linear neutral equations with constant coefficients.
\end{abstract}

1. Introduction. As is well known, it is desirable to find relatively simple equations which may serve as minorant or majorant equations to yield qualitative properties of a given functional differential equation. In this paper, we are concerned with a functional differential equation of the form

$$
(x(t)-P(t) x(t-\tau(t)))^{\prime}+\sum_{j=1}^{m} Q_{j}(t) f_{j}\left(x\left(t-\sigma_{j}(t)\right)\right)=0, \quad t \geq t_{0},
$$

where

(H1) $P, Q_{1}, \ldots, Q_{m}$ are positive continuous functions defined on $\left[t_{0}, \infty\right)$ such that $0 \leq P(t) \leq 1$ on $\left[t_{0}, \infty\right)$,

(H2) $\tau, \sigma_{1}, \ldots, \sigma_{m}$ are continuous functions defined on $\left[t_{0}, \infty\right)$ such that

$$
0<\tau_{*} \leq \tau(t) \leq \tau^{*}, \quad 0<\sigma_{*} \leq \sigma_{j}(t) \leq \sigma^{*} \quad \text { for } j=1, \ldots, m,
$$

and

(H3) $f_{1}, \ldots, f_{m}$ are continuous functions on $\mathbb{R}$ such that $x f_{j}(x)>0$ for $x>0$ and $1 \leq j \leq m$.

More specifically, we are concerned with the question of when this equation has no eventually positive solutions. As a candidate for comparison

\footnotetext{
1991 Mathematics Subject Classification: 34K15, 34C10.

Key words and phrases: neutral differential equations, positive solutions, linearized comparison theorems.
} 
purposes, we will seek a linear equation of the form

$$
(x(t)-p x(t-\tau))^{\prime}+\sum_{j=1}^{m} q_{j} x\left(t-\sigma_{j}\right)=0, \quad t \geq t_{0},
$$

where $p \in[0,1)$, and $q_{1}, \ldots, q_{m}, \tau, \sigma_{1}, \ldots, \sigma_{m}$ are positive constants. Equations of the form (2) have been studied quite extensively (see e.g. [1-7, 10, $11,14]$, while there are only a few studies $[8,9,12,13,15]$ dealing with the existence of eventually positive solutions of (1). Once we can establish a connection between these two equations, information on (1) can be drawn from that concerning (2). Similar investigations have been carried out under the name of linearized oscillation theory (see e.g. [6, Chapter 4]).

As usual (see e.g. [6, p. 5]), a solution of equation (1) is a continuous function defined on an appropriate superset of $\left[t_{0}, \infty\right)$, such that $x(t)-$ $P(t) x(t-\tau(t))$ is continuously differentiable for $t \in\left[t_{0}, \infty\right)$ and $x(t)$ satisfies (1) for $t \in\left[t_{0}, \infty\right)$. A solution of equation (2) is similarly defined. Existence and uniqueness theorems for solutions of either (1) or (2) can be found in [6].

In the next section, we first establish a necessary condition for the existence of an eventually positive solution of (1). This condition turns out to be a necessary and sufficient condition for the existence of an eventually positive solution of a linear equation of the form (2). The desired linearized comparison theorem is then established in section three.

2. Necessary conditions. We first establish a preparatory result relating to the function $y=y(t)$ defined by

$$
y(t)=x(t)-P(t) x(t-\tau(t)),
$$

where $x=x(t)$ is an eventually positive solution of (1).

Lemma 2.1. Suppose $x=x(t)$ is an eventually positive solution of (1). Then the function $y=y(t)$ defined by (3) satisfies $y(t)>0$ and $y^{\prime}(t)<0$ for all large $t$.

Proof. In view of (1), we see that

$$
y^{\prime}(t)=-\sum_{j=1}^{m} Q_{j}(t) f_{j}\left(x\left(t-\sigma_{j}(t)\right)\right)<0
$$

for all large $t$. Thus $y$ is eventually positive or eventually negative. Assume to the contrary that $y(t)<0$ and $y^{\prime}(t)<0$ for all large $t$. Then $y(t) \leq-\alpha<0$ for $t$ greater than or equal to some number $T$, so that

$$
x(t) \leq-\alpha+P(t) x(t-\tau(t)), \quad t \geq T .
$$

We have two cases to consider. First, assume that $x$ is unbounded. Then 
there is a real sequence $\left\{s_{n}\right\}$ which tends to infinity and

$$
x\left(s_{n}\right)=\max _{T \leq t \leq s_{n}} x(t) .
$$

However, in view of the assumption that $0 \leq P(t) \leq 1$, we see that

$$
x\left(s_{n}\right) \leq-\alpha+P\left(s_{n}\right) x\left(s_{n}-\tau\left(s_{n}\right)\right) \leq-\alpha+x\left(s_{n}\right),
$$

which is a contradiction.

Next, assume that $x$ is bounded. Then there is a real sequence $\left\{v_{n}\right\}$ which tends to infinity and $\lim \sup _{n \rightarrow \infty} x\left(v_{n}\right)=L<\infty$. Let $\left\{\xi_{n}\right\}$ be the sequence defined by

$$
x\left(\xi_{n}\right)=\max \left\{x(t) \mid v_{n}-\tau\left(v_{n}\right) \leq t \leq v_{n}\right\} .
$$

Then $\xi_{n} \rightarrow \infty$ and $\limsup _{n \rightarrow \infty} x\left(\xi_{n}\right) \leq L$. Furthermore, we have

$$
x\left(v_{n}\right) \leq-\alpha+P\left(v_{n}\right) x\left(\xi_{n}\right) \leq-\alpha+x\left(\xi_{n}\right)
$$

for all large $n$. Taking superior limits on both sides of this inequality, we see that $L \leq-\alpha+L$, which is also a contradiction. The proof is complete.

By means of Lemma 2.1, we now derive one of our main results related to the existence of an eventually positive solution of (1).

Theorem 2.1. Assume that

$$
f_{j}(x) / x \geq 1 \quad \text { for } x>0 \text { and } j=1, \ldots, m,
$$

and suppose there is a sufficiently large number $T$ such that

(5) $\inf _{t \geq T, \lambda>0}\left\{\frac{1}{\lambda} \sum_{j=1}^{m} Q_{j}(t) e^{\lambda \sigma_{j}(t)}+\frac{1}{\lambda} \sum_{j=1}^{m} Q_{j}(t) P\left(t-\sigma_{j}(t)\right) e^{\lambda \tau\left(t-\sigma_{j}(t)\right)}\right\}>1$.

Then equation (1) cannot have an eventually positive solution.

Proof. Let $x=x(t)$ be an eventually positive solution and let $y=y(t)$ be the function defined by (3). Then by means of Lemma 2.1, we see that $x(t)>0, y(t)>0$ and $y^{\prime}(t)<0$ for $t$ greater than or equal to some $T$. Furthermore, we have

$$
x\left(t-\sigma_{j}(t)\right) \leq f_{j}\left(x\left(t-\sigma_{j}(t)\right)\right),
$$

and

$$
\begin{aligned}
0<y\left(t-\tau\left(t-\sigma_{j}(t)\right)\right) & <y\left(t-\sigma_{j}(t)-\tau\left(t-\sigma_{j}(t)\right)\right) \\
& \leq x\left(t-\sigma_{j}(t)-\tau\left(t-\sigma_{j}(t)\right)\right)
\end{aligned}
$$

for $1 \leq j \leq m$. Define

$$
\lambda(t)=-y^{\prime}(t) / y(t), \quad t \geq T .
$$


Then $\lambda(t)>0$ for $t \geq T$, and

$$
\frac{y(s)}{y(t)}=\exp \left\{\int_{s}^{t} \lambda(s) d s\right\}, \quad s, t \geq T .
$$

In view of (1), we see further that

(6) $\lambda(t)$

$$
\begin{aligned}
= & \sum_{j=1}^{m} \frac{Q_{j}(t) f_{j}\left(x\left(t-\sigma_{j}(t)\right)\right)}{y(t)} \geq \sum_{j=1}^{m} \frac{Q_{j}(t) x\left(t-\sigma_{j}(t)\right)}{y(t)} \\
= & \sum_{j=1}^{m} \frac{Q_{j}(t)\left\{y\left(t-\sigma_{j}(t)\right)+P\left(t-\sigma_{j}(t)\right) x\left(t-\sigma_{j}(t)-\tau\left(t-\sigma_{j}(t)\right)\right)\right\}}{y(t)} \\
\geq & \sum_{j=1}^{m} Q_{j}(t) \exp \left\{\int_{t-\sigma_{j}(t)}^{t} \lambda(s) d s\right\} \\
& +\sum_{j=1}^{m} Q_{j}(t) P\left(t-\sigma_{j}(t)\right) \exp \left\{\int_{t-\tau\left(t-\sigma_{j}(t)\right)}^{t} \lambda(s) d s\right\} .
\end{aligned}
$$

Next, we assert that $\liminf _{t \rightarrow \infty} \lambda(t)>0$. Assume to the contrary that $\liminf _{t \rightarrow \infty} \lambda(t)=0$. Choose a sequence $\left\{s_{n}\right\}$ which tends to infinity and

$$
\lambda\left(s_{n}\right)=\min _{T \leq t \leq s_{n}} \lambda(t) .
$$

Then we see from (6) that

$$
\begin{aligned}
\lambda\left(s_{n}\right) \geq & \sum_{j=1}^{m} Q_{j}\left(s_{n}\right) \exp \left(\lambda\left(s_{n}\right) \sigma_{j}\left(s_{n}\right)\right) \\
& +\sum_{j=1}^{m} Q_{j}\left(s_{n}\right) P\left(s_{n}-\sigma_{j}\left(s_{n}\right)\right) \exp \left(\lambda\left(s_{n}\right) \tau\left(s_{n}-\sigma_{j}\left(s_{n}\right)\right)\right),
\end{aligned}
$$

so that

$$
\begin{aligned}
1 \geq & \inf _{n \geq 1}\left\{\frac{1}{\lambda\left(s_{n}\right)} \sum_{j=1}^{m} Q_{j}\left(s_{n}\right) \exp \left(\lambda\left(s_{n}\right) \sigma_{j}\left(s_{n}\right)\right)\right. \\
& \left.+\frac{1}{\lambda\left(s_{n}\right)} \sum_{j=1}^{m} Q_{j}\left(s_{n}\right) P\left(s_{n}-\sigma_{j}\left(s_{n}\right)\right) \exp \left(\lambda\left(s_{n}\right) \tau\left(s_{n}-\sigma_{j}\left(s_{n}\right)\right)\right)\right\}
\end{aligned}
$$

contrary to our assumption (5).

Next, we assert that $\liminf _{t \rightarrow \infty} \lambda(t)<\infty$. Assume to the contrary that $\liminf _{t \rightarrow \infty} \lambda(t)=\infty$. Pick an arbitrary positive number $c$. Then in view of 
(5), we see that

$1<\left\{\frac{1}{c} \sum_{j=1}^{m} Q_{j}(t) \exp \left(c \sigma_{j}(t)\right)+\frac{1}{c} \sum_{j=1}^{m} Q_{j}(t) P\left(t-\sigma_{j}(t)\right) \exp \left(c \tau\left(t-\sigma_{j}(t)\right)\right)\right\}$

which implies

$$
\left\{\sum_{j=1}^{m} Q_{j}(t)\left(1+P\left(t-\sigma_{j}(t)\right)\right)\right\} \geq c \exp \left(-c \max \left\{\sigma^{*}, \tau^{*}\right\}\right)>0,
$$

and thus

$$
\begin{aligned}
\liminf _{t \rightarrow \infty} \int_{t-\min \left\{\sigma_{*}, \tau_{*}\right\}}^{t}\left\{\sum_{j=1}^{m} Q_{j}(t)\left(1+P\left(t-\sigma_{j}(t)\right)\right)\right\} d t \\
\geq c \min \left\{\sigma_{*}, \tau_{*}\right\} \exp \left(-c \max \left\{\sigma^{*}, \tau^{*}\right\}\right)>0 .
\end{aligned}
$$

On the other hand, in view of (6), we have

$$
\lambda(t) \geq\left\{\sum_{j=1}^{m} Q_{j}(t)\left(1+P\left(t-\sigma_{j}(t)\right)\right)\right\} \exp \left\{\int_{t-\min \left\{\sigma_{*}, \tau_{*}\right\}}^{t} \lambda(s) d s\right\} .
$$

Thus, by invoking Lemma 2.1 in [5], we see that

$$
\liminf _{t \rightarrow \infty} \int_{t-\min \left\{\sigma_{*}, \tau_{*}\right\}}^{t} \lambda(s) d s<\infty
$$

contrary to our assumption that $\liminf _{t \rightarrow \infty} \lambda(t)=\infty$.

To complete our proof, let us denote $\liminf _{t \rightarrow \infty} \lambda(t)$ by $\lambda_{*}$. Also let $\eta>1$ be an arbitrary number such that

$$
\inf _{t \geq T, \lambda>0}\left\{\frac{1}{\lambda} \sum_{j=1}^{m} Q_{j}(t) e^{\lambda \sigma_{j}(t)}+\frac{1}{\lambda} \sum_{j=1}^{m} Q_{j}(t) P\left(t-\sigma_{j}(t)\right) e^{\lambda \tau\left(t-\sigma_{j}(t)\right)}\right\}>\eta .
$$

For sufficiently large $t$, since

$$
\eta \lambda\left(t-\tau\left(t-\sigma_{j}(t)\right)\right)>\lambda_{*} \text { and } \eta \lambda\left(t-\sigma_{j}(t)\right)>\lambda_{*}
$$

for $1 \leq j \leq m$, we see from (6) that

$$
\begin{aligned}
\lambda_{*} \geq & \inf _{s \geq T}\left\{\sum_{j=1}^{m} Q_{j}(s) \exp \left(\frac{\lambda_{*}}{\eta} \sigma_{j}(s)\right)\right. \\
& \left.+\sum_{j=1}^{m} Q_{j}(s) P\left(s-\sigma_{j}(s)\right) \exp \left(\frac{\lambda_{*}}{\eta} \tau\left(s-\sigma_{j}(s)\right)\right)\right\} .
\end{aligned}
$$


After rewriting this inequality, we see that

$$
\begin{aligned}
\eta \geq \inf _{s \geq T}\left\{\frac{\eta}{\lambda_{*}} \sum_{j=1}^{m} Q_{j}(s) \exp \left(\frac{\lambda_{*}}{\eta} \sigma_{j}(s)\right)\right. \\
\left.+\frac{\eta}{\lambda_{*}} \sum_{j=1}^{m} Q_{j}(s) P\left(s-\sigma_{j}(s)\right) \exp \left(\frac{\lambda_{*}}{\eta} \tau\left(s-\sigma_{j}(s)\right)\right)\right\},
\end{aligned}
$$

contrary to our assumption (7). The proof is complete.

As an immediate corollary, note that

$$
\min _{\lambda>0} e^{\lambda \xi} / \lambda=\xi e, \quad \xi>0,
$$

which implies that for $\lambda>0$,

$$
\begin{aligned}
\frac{1}{\lambda} \sum_{j=1}^{m} Q_{j}(t) e^{\lambda \sigma_{j}(t)}+ & \frac{1}{\lambda} \sum_{j=1}^{m} Q_{j}(t) P\left(t-\sigma_{j}(t)\right) e^{\lambda \tau\left(t-\sigma_{j}(t)\right)} \\
& \geq \sum_{j=1}^{m} Q_{j}(t) e\left\{\sigma_{j}(t)+P\left(t-\sigma_{j}(t)\right) \tau\left(t-\sigma_{j}(t)\right)\right\} .
\end{aligned}
$$

Thus, if

$$
\liminf _{t \rightarrow \infty} \sum_{j=1}^{m} Q_{j}(t)\left\{\sigma_{j}(t)+P\left(t-\sigma_{j}(t)\right) \tau\left(t-\sigma_{j}(t)\right)\right\}>\frac{1}{e},
$$

then (1) cannot have an eventually positive solution.

There are two variants of the above theorem. The first one assumes the additional condition that $\tau(t) \equiv \tau, \sigma_{j}(t) \equiv \sigma_{j}$ and $f_{j}(x) \leq \delta_{j} x$ for $x>0$.

Theorem 2.2. Assume that $\tau(t) \equiv \tau, \sigma_{j}(t) \equiv \sigma_{j}$ for $1 \leq j \leq m$, that

$$
1 \leq f_{j}(x) / x \leq \delta_{j} \quad \text { for } x>0 \text { and } 1 \leq j \leq m,
$$

and that there is a number $T$ such that

$$
\inf _{t \geq T, \lambda>0}\left\{\frac{1}{\lambda} \sum_{j=1}^{m} Q_{j}(t) e^{\lambda \sigma_{j}}+\frac{\Gamma(t)}{\delta} e^{\lambda \tau}\right\}>1,
$$

where $\delta=\max _{1 \leq j \leq m} \delta_{j}$ and

$$
\Gamma(t)=\min _{1 \leq j \leq m} \frac{Q_{j}(t) P\left(t-\sigma_{j}\right)}{Q_{j}(t-\tau)} .
$$

Then (1) cannot have an eventually positive solution. 
Proof. We only need to note that (6) now changes to

$$
\begin{aligned}
\lambda(t) \geq & \sum_{j=1}^{m} Q_{j}(t) \exp \left\{\int_{t-\sigma_{j}}^{t} \lambda(s) d s\right\} \\
& +\sum_{j=1}^{m} \frac{\left.Q_{j}(t) P\left(t-\sigma_{j}\right) x\left(t-\sigma_{j}-\tau\right)\right)}{y(t)},
\end{aligned}
$$

and the second sum $S(t)$ in the above inequality is equal to

$$
\sum_{j=1}^{m}\left\{\frac{Q_{j}(t) P\left(t-\sigma_{j}\right)}{Q_{j}(t-\tau)} \cdot \frac{Q_{j}(t-\tau) x\left(t-\sigma_{j}-\tau\right)}{y(t)}\right\}
$$

so that

$$
\begin{aligned}
S(t) & \geq \Gamma(t) \sum_{j=1}^{m} \frac{Q_{j}(t-\tau) f_{j}\left(x\left(t-\sigma_{j}-\tau\right)\right)}{y(t) \delta_{j}} \\
& \geq \frac{\Gamma(t)}{\delta y(t)}\left\{-y^{\prime}(t-\tau)\right\}=\frac{\Gamma(t)}{\delta} \lambda(t-\tau) \frac{y(t-\tau)}{y(t)} \\
& =\frac{\Gamma(t)}{\delta} \lambda(t-\tau) \exp \left\{\int_{t-\tau}^{t} \lambda(s) d s\right\} .
\end{aligned}
$$

The rest of the proof is similar to that of Theorem 2.1, and is thus omitted.

We remark that the condition (8) is needed in several inequalities in the proof. If we make the additional assumption that any eventually positive solution $x=x(t)$ also converges to zero, then condition (8) can be weakened to requiring

$$
1 \leq \liminf _{x \rightarrow 0} \frac{f_{j}(x)}{x} \leq \limsup _{x \rightarrow 0} \frac{f_{j}(x)}{x} \leq \delta_{j}, \quad 1 \leq j \leq m .
$$

We only need to modify slightly several arguments in the proof of the above theorem to yield the following variant.

Theorem 2.3. Assume that $\tau(t) \equiv \tau, \sigma_{j}(t) \equiv \sigma_{j}$ for $1 \leq j \leq m$, that $(10)$ holds and that there is a number $T$ such that (9) holds where $\delta$ and $\Gamma(t)$ are defined in Theorem 2.2. Then (1) cannot have an eventually positive solution which converges to zero.

It is not difficult to impose conditions such that all eventually positive solutions of (1) converge to zero. For instance, the following assertion holds: Assume that

$$
\lim _{t \rightarrow \infty} P(t)=p \in[0,1),
$$


and that one of the functions $Q_{1}, \ldots, Q_{m}$, say $Q_{j^{*}}$, satisfies

$$
\int_{t_{0}}^{\infty} Q_{j^{*}}(s) d s=\infty
$$

If $x=x(t)$ is an eventually positive solution of (1), then $\lim _{t \rightarrow \infty} x(t)$ exists and equals 0 .

Indeed, let $x=x(t)$ be an eventually positive solution of (1). Then in view of Lemma 2.1, the function $y=y(t)$ defined by (3) satisfies $y(t)>0$ and $y^{\prime}(t)<0$ for all large $t$. Thus we have

$$
0<y(t) \leq y\left(t-\sigma_{j^{*}}(t)\right) \leq x\left(t-\sigma_{j^{*}}(t)\right) \leq f_{j^{*}}\left(x\left(t-\sigma_{j^{*}}(t)\right)\right)
$$

for $t$ greater than or equal to some number $T$. Without loss of any generality, we may assume that $P(t)<p^{\prime}$ for $t \geq T$, where $p^{\prime} \in(p, 1)$. Employing these facts, we then deduce from (1) that

$$
y^{\prime}(t)+Q_{j^{*}}(t) y(t) \leq 0, \quad t \geq T .
$$

After integrating, we have

$$
y(t) \exp \int_{T}^{t} Q_{j^{*}}(s) d s \leq y(T), \quad t \geq T,
$$

which implies

$$
\begin{aligned}
x(t) & \leq P(t) x(t-\tau(t))+y(T) \exp \left\{-\int_{T}^{t} Q_{j^{*}}(s) d s\right\} \\
& <p^{\prime} x(t-\tau(t))+y(T) \exp \left\{-\int_{T}^{t} Q_{j^{*}}(s) d s\right\} .
\end{aligned}
$$

If $x$ is not bounded, then there is a sequence $\left\{s_{n}\right\}$ which tends to infinity and (4) holds. Thus,

$$
\begin{aligned}
x\left(s_{n}\right) & <p^{\prime} x\left(s_{n}-\tau\left(s_{n}\right)\right)+y(T) \exp \left\{-\int_{T}^{s_{n}} Q_{j^{*}}(s) d s\right\} \\
& \leq p^{\prime} x\left(s_{n}\right)+y(T) \exp \left\{-\int_{T}^{s_{n}} Q_{j^{*}}(s) d s\right\},
\end{aligned}
$$

which implies

$$
x\left(s_{n}\right)<\frac{y(T)}{1-p^{\prime}} \exp \left\{-\int_{T}^{s_{n}} Q_{j^{*}}(s) d s\right\}
$$

for all large $n$. This is impossible as can be seen by taking limits on both sides. We have thus shown that $x$ is bounded. 
Next we show that $x$ has a limit. Indeed, let $\left\{v_{n}\right\}$ be a divergent sequence such that $\lim \sup _{t \rightarrow \infty} x(t)=\lim _{n \rightarrow \infty} x\left(v_{n}\right)$. Then in view of (3), we see that $\limsup _{t \rightarrow \infty} x(t)=\lim _{t \rightarrow \infty} y(t)+p \lim _{n \rightarrow \infty} x\left(v_{n}-\tau\left(v_{n}\right)\right) \leq \lim _{t \rightarrow \infty} y(t)+p \limsup _{t \rightarrow \infty} x(t)$,

which implies

Similarly, we have

$$
\limsup _{t \rightarrow \infty} x(t) \leq \frac{\lim _{t \rightarrow \infty} y(t)}{1-p}
$$

$$
\frac{\lim _{t \rightarrow \infty} y(t)}{1-p} \leq \liminf _{t \rightarrow \infty} x(t) .
$$

Finally, if $\lim _{t \rightarrow \infty} x(t)=\alpha>0$, then $0<\alpha / 2 \leq x(t)$ for $t$ greater than or equal to some number $t_{1}$. Since $f(t) \geq t$ for $t>0$, we see that

$$
f_{j^{*}}\left(x\left(t-\sigma_{j}(t)\right) \geq x\left(t-\sigma_{j}(t)\right) \geq \alpha / 2\right.
$$

for $t$ greater than or equal to some number $t_{2} \geq t_{1}$. Thus by means of (1), we have

$$
y^{\prime}(t)=-\sum_{j=1}^{m} Q_{j}(t) f_{j}\left(x\left(t-\sigma_{j}(t)\right)\right) \leq-Q_{j^{*}}(t) \alpha / 2, \quad t \geq t_{2} .
$$

By integrating the above inequality from $t_{2}$ to $\infty$, we conclude from (12) that $\lim _{t \rightarrow \infty} y(t)=-\infty$. This contradicts the conclusion of Lemma 2.1.

3. Linearized comparison theorem. In this section, we will exhibit a connection between equation (1) and an appropriate linear equation of the form (2). Recall first that the assumptions that $p \in[0,1)$, and $q_{1}, \ldots$ $\ldots, q_{m}, \tau, \sigma_{1}, \ldots, \sigma_{m}>0$ have been made. Next, we establish two properties of (2) which are needed for our linearized comparison theorem.

Theorem 3.1. If the condition

$$
F(\lambda) \equiv-\lambda+\lambda p e^{\lambda \tau}+\sum_{j=1}^{m} q_{j} e^{\lambda \sigma_{j}}>0
$$

holds for all $\lambda>0$, then (2) cannot have an eventually positive solution. The converse also holds.

Proof. The first statement follows from Theorem 2.2 by taking $f_{j}(x)=$ $x$ and $\delta_{j}=1$ for $1 \leq j \leq m$. To see that the converse holds, suppose there is a positive number $\lambda^{*}$ such that $F\left(\lambda^{*}\right) \leq 0$. Note that $F(+\infty)=+\infty$, thus there is a number $\xi \in\left[\lambda^{*}, \infty\right)$ such that $F(\xi)=0$. It is then easily verified that the function $x=x(t)$ defined by $x(t)=\exp (-\xi t)$ is an eventually positive solution of (13).

Next, we establish a theorem on continuous dependence on parameters for linear equations of the form (2). 
TheOREM 3.2. Suppose $p>0$ and that (2) does not have any eventually positive solutions. Then there is a positive number $\mu<\min \left\{p, q_{1}, \ldots, q_{m}\right\}$ such that for every $\varepsilon \in[0, \mu]$, the equation

$$
(x(t)-(p-\varepsilon) x(t-\tau))^{\prime}+\sum_{j=1}^{m}\left(q_{j}-\varepsilon\right) x\left(t-\sigma_{j}\right)=0
$$

cannot have any eventually positive solutions either.

Proof. By means of Theorem 3.1, we see that the function $F=F(\lambda)$ defined by (13) satisfies $F(\lambda)>0$ for $\lambda>0$. Furthermore, it is easily verified that $\lim _{\lambda \rightarrow 0} F(\lambda)>0, F(+\infty)=+\infty$ and $F^{\prime \prime}(\lambda)>0$ for $\lambda>0$. Thus $F(\lambda) \geq c>0$ for $\lambda>0$.

Define

$$
F(\lambda, \theta)=-\lambda+\lambda(p-\theta) e^{\lambda \tau}+\sum_{j=1}^{m}\left(q_{j}-\theta\right) e^{\lambda \sigma_{j}}, \quad \lambda>0,-\infty<\theta<\infty .
$$

Note that $F(\lambda, 0)=F(\lambda) \geq c>0$ for $\lambda>0$, that $F_{\theta}(\lambda, \theta)<0$ and that $F(+\infty, \theta)=+\infty$ for $0<\theta<\min \left\{p, q_{1}, \ldots, q_{m}\right\}$. Therefore, since $F(\lambda, \theta)$ is continuous in $\lambda$ and $\theta$, it is not difficult to find a positive number $\mu<\min \left\{p, q_{1}, \ldots, q_{n}\right\}$ such that $F(\lambda, \mu)>0$ for $\lambda>0$. Next, since $F(\lambda, \theta)$ is decreasing in $\theta$ for each fixed $\lambda$, we see that $F(\lambda, \theta)>0$ for each $\lambda>0$ and $\theta \in[0, \mu]$. The proof is complete.

The same idea can be employed to show the following variant of Theorem 3.2: Suppose $p=0$ and that (2) does not have any eventually positive solutions. Then there is a positive number $\mu<\min \left\{q_{1}, \ldots, q_{m}\right\}$ such that for every $\varepsilon \in[0, \mu]$, the equation

$$
x^{\prime}(t)+\sum_{j=1}^{m}\left(q_{j}-\varepsilon\right) x\left(t-\sigma_{j}\right)=0
$$

cannot have any eventually positive solutions either.

We now state and prove our final linearized comparison theorem. We need the assumptions that

$$
\begin{gathered}
\lim _{t \rightarrow \infty} P(t)=p \in[0,1), \\
\lim _{t \rightarrow \infty} Q_{j}(t)=q_{j}, \quad 1 \leq j \leq m,
\end{gathered}
$$

and

$$
\lim _{x \rightarrow 0} \frac{f_{j}(x)}{x}=1, \quad 1 \leq j \leq m .
$$

Theorem 3.3. Assume that (17)-(19) hold. Assume further that $\tau(t) \equiv$ $\tau$, and $\sigma_{j}(t) \geq \sigma_{j}$ for $1 \leq j \leq m$ for $t \geq t_{0}$. If (2) does not have an eventually positive solution, then neither has (1). 
Proof. We assume that $0<p<1$. Since (2) does not have any eventually positive solutions, by Theorem 3.2 , there is a positive number $\mu<\min \left\{p, q_{1}, \ldots, q_{m}\right\}$ such that for every $\varepsilon \in[0, \mu]$, the equation (14) cannot have any eventually positive solutions either. Thus, by Theorem 3.1,

$$
-\lambda+\lambda(p-\varepsilon) e^{\lambda \tau}+\sum_{j=1}^{m}\left(q_{j}-\varepsilon\right) e^{\lambda \sigma_{j}}>0, \quad \lambda>0, \varepsilon \in[0, \mu] .
$$

Note further that (18) and $\lim _{t \rightarrow \infty} \Gamma(t)=p$ imply respectively that $Q_{j}(t) \geq$ $q_{j}-\varepsilon$ and $\Gamma(t) \geq p-\varepsilon$ for all large $t$. Thus,

$$
\frac{1}{\lambda} \sum_{j=1}^{m} Q_{j}(t) e^{\lambda \sigma_{j}(t)}+\Gamma(t) e^{\lambda \tau} \geq \frac{1}{\lambda} \sum_{j=1}^{m}\left(q_{j}-\varepsilon\right) e^{\lambda \sigma_{j}}+(p-\varepsilon) e^{\lambda \tau}>1
$$

for all $\lambda>0$ and all large $t$. By Theorem 2.2, (1) cannot have any eventually positive solutions.

The case where $p=0$ is similarly proved.

4. Examples and remarks. Consider the equation

$$
\begin{aligned}
(x(t) & \left.-\frac{1}{2} x\left(t-\frac{\pi}{2}\right)\right)^{\prime}+\frac{1}{4} x\left(t-\frac{\pi}{2}\right) \\
& +\frac{1}{2} x\left(t-\frac{5 \pi}{2}\right)+\frac{1}{4} x(t-4 \pi)+\frac{1}{2} x\left(t-\frac{9 \pi}{2}\right)=0, \quad t>5 \pi .
\end{aligned}
$$

It is easy to verify that $x(t)=\sin t$ is a solution which is neither eventually positive nor eventually negative. Actually, we may also verify that the conditions in Theorem 3.1 hold. Thus this equation cannot have any eventually positive solutions.

As another example, consider the equation

$$
\begin{aligned}
\left(x(t)-\left(\frac{1}{2}+\frac{1}{t^{2}}\right)\right. & x(t-2-\cos t))^{\prime} \\
+ & \frac{1}{2} x(t-2+\sin t)+t^{2} x(t-2-\sin t)=0, \quad t>3 .
\end{aligned}
$$

It is easy to verify that the conditions in Theorem 2.2 hold. Thus this equation cannot have any eventually positive solutions either.

We remark that in case the hypothesis (H3) in Section 1 is replaced by requiring each of the functions $f_{j}(x)$ in (1) to satisfy the condition $x f_{j}(x)<0$ for $x<0$, then it is not hard to see that a dual statement of Theorem 2.1 holds for eventually negative solutions. The subsequent results in the previous sections may also be restated for eventually negative solutions. Consequently, oscillation criteria can be obtained by replacing (H3) with $x f_{j}(x)>0$ for $x \neq 0$ for each $j$. This procedure is quite standard and can be found in various references. 
The idea behind the proof of Theorem 2.1 is not new. Indeed, similar ideas can be found in $[9,11,15]$ and elsewhere. Our proofs contain various technical differences, however. It is worth mentioning that Yan [13] has approached similar problems by alternate means. Later Yan's idea was used again in [4]. It seems that Yan's approach is also applicable to our equation (1), but the details are far from complete.

Finally, we remark that equation (1) is more general than some of the equations which are studied in $[2,4,7,11]$. Thus our results improve, generalize and/or overlap with some of the results contained in these studies (see e.g. [2, Theorem 3.4], [4, Theorem 1] or [11, Theorem 1]).

\section{References}

[1] M. P. Chen, J. S. Yu and L. H. Huang, Oscillations of first order neutral differential equations with variable coefficients, J. Math. Anal. Appl. 185 (1994), 288-301.

[2] K. Gopalsamy and B. G. Zhang, Oscillation and nonoscillation in first order neutral differential equations, ibid. 151 (1990), 42-57.

[3] M. K. Grammatikopoulous, Y. G. Sficas and I. P. Stavroulakis, Necessary and sufficient conditions for oscillations of neutral equations with several coefficients, J. Differential Equations 76 (1988), 294-311.

[4] E. A. Grove, M. R. S. Kulenovic and G. Ladas, Sufficient conditions for oscillation and nonoscillation of neutral equations, ibid. 68 (1987), 373-382.

[5] I. Gyori, On the oscillatory behaviour of solutions of certain nonlinear and linear delay differential equations, Nonlinear Anal. 8 (1984), 429-439.

[6] I. Gyori and G. Ladas, Oscillation Theory of Delay Differential Equations with Applications, Oxford University Press, 1991.

[7] G. Ladas and Y. G. Sficas, Oscillations of neutral delay differential equations, Canad. Math. Bull. 29 (1986), 438-445.

[8] W. Lu, Existence of nonoscillatory solutions of first order nonlinear neutral equations, J. Austral. Math. Soc. Ser. B 32 (1990), 180-192.

[9] -, Nonoscillation and oscillation for first order nonlinear equations, Funkcial. Ekvac. 37 (1994), 383-394.

[10] R. Olah, Oscillation of differential equation of neutral type, Hiroshima Math. J. 25 (1995), 1-10.

[11] C. Qian, G. Ladas, B. G. Zhang and T. Zhao, Sufficient conditions for oscillation and existence of positive solutions, Appl. Anal. 35 (1990), 187-194.

[12] J. H. Shen and Z. C. Wang, Oscillation and nonoscillation for a class of nonlinear neutral differential equations, Differential Equations Dynam. Systems 2 (1994), 347360 .

[13] J. Yan, Oscillation of solutions of first order delay differential equations, Nonlinear Anal. 11 (1987), 1279-1287.

[14] J. S. Yu and Z. Wang, A linearized oscillation result for neutral delay differential equations, Math. Nachr. 163 (1993), 101-107. 
[15] B. G. Zhang and J. S. Y u, Oscillation and nonoscillation for neutral differential equations, J. Math. Anal. Appl. 172 (1993), 11-23.

Department of Mathematics

Northeast Heavy Machinery Institute

Qiqihar, Heilongjiang

161042 P.R. China
Department of Mathematics

Tsing Hua University

Hsinchu, Taiwan, 30043 R.O.C.

Reçu par la Rédaction le 8.11.1995 